\title{
A method for calibrating an eye-monitoring system for use with infants
}

\author{
CHRISTOPHER M. HARRIS, LOUISE HAINLINE, and ISRAEL ABRAMOV \\ Brooklyn College, City University of New York, Brooklyn, New York 11210
}

\begin{abstract}
An algorithm for calibrating data from an infrared corneal reflection eye movement monitor is described. The algorithm is designed for use with infants and other noninstructable subjects.
\end{abstract}

A serious problem encountered in eye movement monitors that rely on Purkinje images to estimate a subject's point of regard (see, e.g., Young \& Sheena, 1975 ) is relating derived points of regard to actual points of fixation. Calibration of such instruments, which are vulnerable to noise perturbations and nonlinearities, is desirable for maximum precision. Large errors in estimating point of regard on the target plane can arise from small instrument biases or random noise. Errors can also arise from individual differences in corneal aspherism, astigmatism, and misalignment of the optical elements (Carpenter, 1980) can give rise to erroneous estimates of the geometrical optical axis used in the calculation of eye position. In addition, there is no certainty that the geometric optic axis is indeed used as the visual axis by the infant. Slater and Findlay (1972) have argued that for infants, and to a lesser extent for adults, the fovea is temporal to the point at which the optic axis intersects the retina; such a displacement might cause fixation estimates to be systematically biased to the temporal side. Also, if the critical area of the retina used for central vision is off the optic axis, paraxial imaging would not apply, giving rise to further aberrations due to off-axis optics. In general, these optical aberrations will give rise to nonlinear and/or anisotropic errors that may adversely affect even the relative data used for calculating, for example, relative velocities or accelerations. The nature and magnitudes of many of these optical errors show individual variability. They may be a greater problem for infant than for adult subjects. Finally, these problems can be exacerbated by head movements.

There are methods of overcoming these errors in measuring a subject's point of regard. The most common approach is to instruct the subject to fixate a series of spatially separated points and have the operator adjust the gain of the recording hardware or make a software correction to compensate for the particular subject's

The research reported here was supported by Grant GB43888 from the National Science Foundation, Grant HD08706 from the National Institutes of Health, Grant 12220 from the PSCBHE Faculty Research Awards Program of the City University of New York, and Grant 12-19 from the March of Dimes Birth Defects Foundation. We thank Lawrence Sirovich for his valuable assistance. errors. However, this approach cannot readily be used with noninstructable subjects. This paper describes a procedure especially designed to calibrate estimates of eye position from infant subjects (or any other noninstructable subject) when head movements have been minimized.

A basic problem is persuading infants to fixate a set of calibration points in as short a time as possible so that their attention span is not exhausted. Unfortunately, this precludes the usual adult procedure of on-line correction. Consequently, an algorithm has been developed to obtain correction parameters from calibration data and apply them to the experimental data off-line. While it is likely that normal adults use the fovea to fixate and direct eye movements, there is reason to suspect that the young infant, because of retinal immaturity, may have a fovea that is either nonfunctioning or functionally immature (Bronson, 1974; Mann, 1964; Abramov, Gordon, Hendrickson, Hainline, Dobson, \& LaBossiere, Note 1). The procedure described here assumes that the area of the retina directing the eye is used consistently across fixations; this retinal area does not have to be the fovea. If this assumption is violated (for example, if "fixations" are made with an annulus around the central fovea), the residual error after calibration would include error proportional to the diameter of the annulus.

The apparatus and general experimental configuration used for infant subjects are described elsewhere (Hainline, 1981). Since in our experience infants will not tolerate rigid head restraint, an experimenter holds the infant over the shoulder and positions the subject's head to keep the image of the eye within the narrow field of view of the system's TV camera; our system has some hardware compensation for head movements and limits permissible head translations to about $\pm 1 \mathrm{~cm}$ on all three axes. Given a viewing distance of $50 \mathrm{~cm}$, this is a reasonable limitation.

The infant is presented a sequence of up to nine rear-projected 1-deg calibration points in succession (see Figure 1). These points are arranged in a circle, the diameter of which subtends a visual angle of $20 \mathrm{deg}$. Each point is flickered to get the infant's attention. Whenever the infant is judged by the operator to be fixating the calibration point, the on-line computer data collection routine is enabled. ${ }^{1}$ The operator makes this 


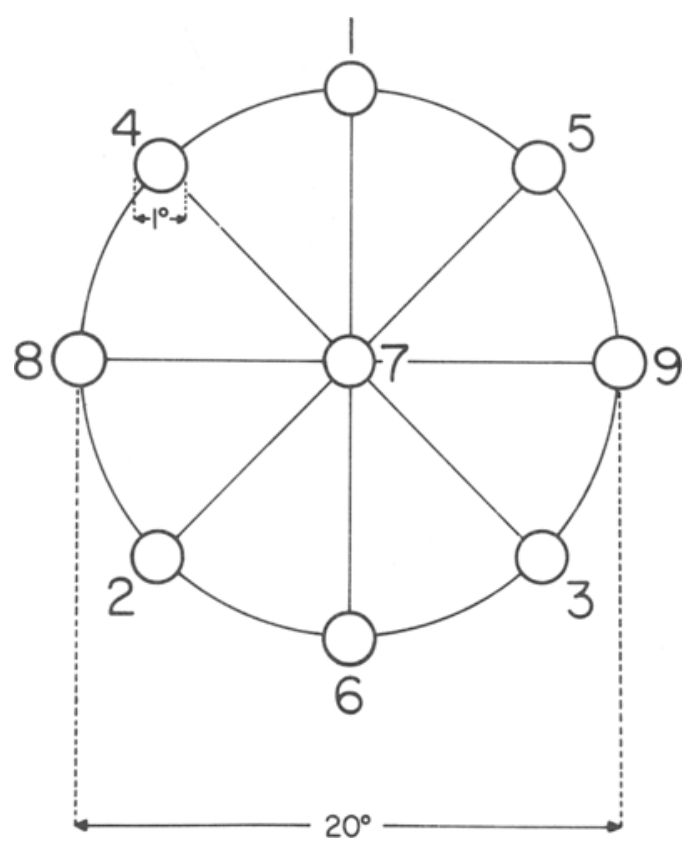

Figure 1. The spatial array of calibration points presented to the subject one at a time. The numbers next to the points represent a preferred order of presentation.

judgment by noting the position of the cross hairs (see Hainline, 1981) that display the uncalibrated estimate of point of regard on a TV monitor that also shows the visual stimulus. In our apparatus, the $X, Y$ coordinates of these points of regard are obtained every $16.7 \mathrm{msec}$, and up to $10 \mathrm{sec}$ of data are collected for a given calibration stimulus point. This procedure is continued for each calibration point in turn until either a full set is obtained or the infant ceases to cooperate. The data are saved on a mass storage device for later analysis. The advantage of an off-line procedure lies in the fact that the calibration data can be collected at any time before, during, or, as in our setting, after gathering experimental data, thus permitting a more flexible experimental schedule. There is an inherent assumption of stationarity, in that it is assumed that the distribution of errors, whether instrumental or physiological in origin, remains constant over the time between gathering the experimental and calibration data. Naturally, this assumption is weakened if the delay is excessive.

The remainder of the calibration procedure is the application of a two-step algorithm that is now described. First, because we have no a priori information as to which portions of the data are attempts to fixate a particular calibration point and which are nonfixational, we must extract a best estimate of the subject's point of fixation from the data for each calibration point. The spatial displacements between these estimates and the corresponding calibration points are then calculated to give a set of error vectors. Second, using these error vectors, some overall correction is determined; using standard least squares techniques, a two-dimensional polynomial is calculated that can be used to correct the subject's experimental data.

\section{THE CENTRAL TENDENCY OF FIXATION}

Consider a simulated but typical distribution of eye positions on the target plane, containing the calibration point being viewed (see Figure 2a). From this distribution, some estimate of the point of regard must be made; that is, some measure of central tendency must be found that will be insensitive to highly variant nonfixational data points representing saccades, "homing in" on the stimulus, and so on. We assume the attempts to fixate the calibration point to be distributed symmetrically about some mean $\bar{F}$ (with variance $S_{F}^{2}$ ); we also assume $\bar{F}$ to be the best estimate of the point of regard. We may also consider the nonfixational, confounding points as belonging to some other distribution with a mean $\bar{C}$ (and variance $S_{C}^{2}$ ). The problem can then be restated as finding the fixation mean, $\overline{\mathrm{F}}$, when the fixation distribution is confounded by another unknown distribution.
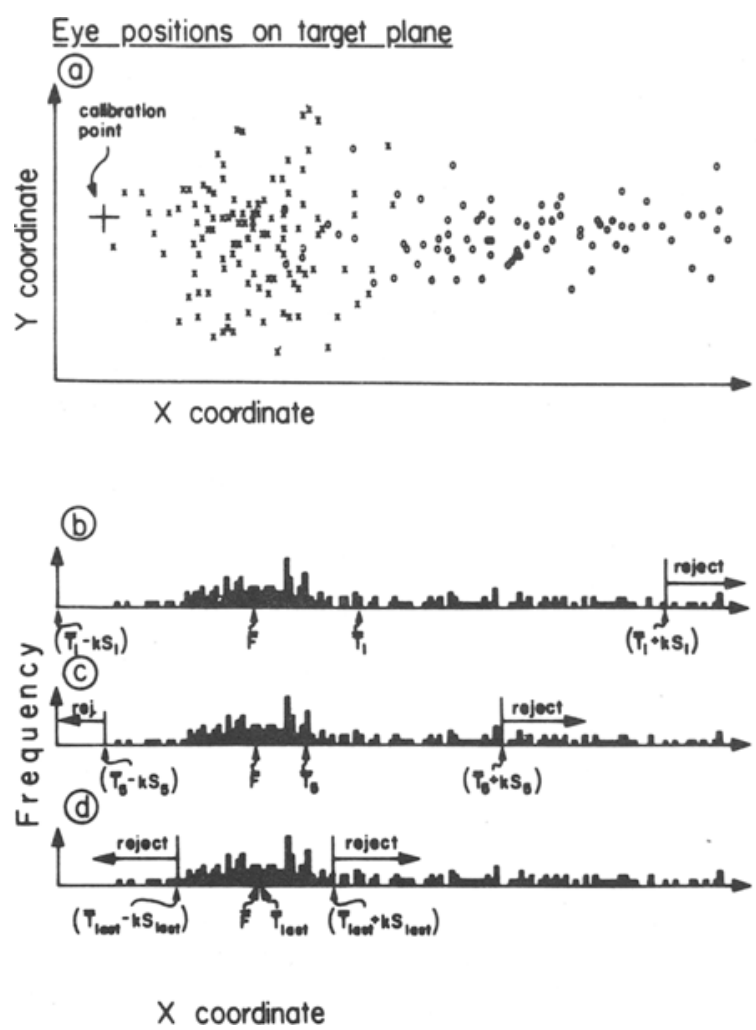

Figure 2. (a) A simulation of eye positions for a subject viewing a calibration point. Xs indicate fixational data points; $O$ s indicate confounding data points. (b) $A$ histogram of all the data points in Figure $2 a$ in the $X$ direction. $\bar{F}$ indicates the fixational mean. $\bar{T}_{1}$ represents the mean from the first iteration. The points $\left(\bar{T}_{1} \pm k S_{1}\right)$ indicate the rejection boundaries of the first iteration. (c) The result of the fifth iteration for the distribution in Figure 2b. (d) The result of the final iteration for the same distribution showing the final estimate, $\overline{\mathrm{T}}_{\text {last }}$. 
In the presence of this confounding, the simple overall mean, $\bar{T}$, will not suffice, since $\bar{T}=f \bar{F}+c \bar{C}$, where $\mathrm{f}=$ proportion of fixational points, $\mathrm{c}=$ proportion of confounding points, and $\mathrm{f}+\mathrm{c}=1$.

Of course, if the confounding distribution $\mathrm{C}$ is symmetrical about $\overline{\mathrm{F}}$, then $\overline{\mathrm{T}}=\overline{\mathrm{F}}=\overline{\mathrm{C}}$ and our problem disappears, but unfortunately, this does not usually occur with infant data. An alternative measure of central tendency might be the median, since it is reasonably insensitive to highly variant points. Our objection to the median is twofold: It has no theoretical underpinning with regard to least squares error, and its use also requires extra a priori assumptions that we are not prepared to make. The following procedure is therefore used.

A simple mean and variance are calculated from all of the obtained data points for a particular calibration stimulus. Any points lying outside a preset number of standard deviations (SDs) are rejected, and a new mean and variance are calculated from the remaining nonrejected points. The original sample is analyzed again and points lying outside the preset number of new SDs from the new mean are rejected, yielding the latest mean and variance. This procedure is continued until the number of rejected points is constant from one iteration to the next. We then take this last mean as the most representative coordinate of the subject's point of fixation, $\bar{F}$. See Figure 2 for a one-dimensional example of samples from this progression. Since the data are actually two-dimensional, both $\mathrm{X}$ and $\mathrm{Y}$ means and SDs are calculated on each iteration and then combined to form an area of acceptance (nonrejection).

Unfortunately, the first part of the algorithm does not lend itself to a simple mathematical treatment, but it can be explained easily on an intuitive level. As an example, consider the points in Figure $2 \mathrm{a}$, collapsed onto the $\mathrm{X}$ axis as shown in Figure $2 b$. Let us assume that the data points (denoted by Xs) associated with the attempt to fixate the calibration point belong to a normal distribution and the confounding nonfixation points (denoted by Os) belong to a rectangular distribution. On the first iteration, any points lying outside the region $\bar{T}_{1} \pm \mathrm{kS}_{1}$ (where $\mathrm{k}$ is the preset criterion) will be rejected; however, if the variance of the confounding points is greater than that of the fixational points, a higher proportion of the confounding points will be rejected and the mean of the remaining points will shift toward the fixation mean $\overline{\mathrm{F}}$. This progression toward the fixation mean will continue until the proportions of fixational and confounding points rejected become equal and convergence has been reached (Figgures $2 \mathrm{c}$ and $2 \mathrm{~d}$ ). However, it is impossible to detect this equalization without a priori knowledge of the two distributions; the criterion we employ is to stop iteration when the number of rejected points becomes constant; this is essentially equivalent to stopping once the mean of the remaining distribution becomes constant. The final distribution of nonrejected points will, in general, have some residual asymmetry so that $\overline{\mathrm{T}}_{\text {last }} \neq \overline{\mathrm{F}}$, but practically, the final estimate is close to $\bar{F}$. The final point of convergence, $\bar{T}_{\text {last }}$, and indeed the conditions for convergence, are a complex combination of the shapes of the fixation and confounding distributions, of the separations of their means, of the proportions $f$ and $c$, and of the ratio of the variances $S_{F}^{2}$ to $S_{C}^{2}$. In general, convergence will occur toward the fixation means if: (1) the proportion of fixational points is greater than the proportion of the confounding points, $f>c$, and (2) the variance of the fixational points is less than the variance of the confounding points, $\mathrm{S}_{\mathrm{f}}^{2}<\mathrm{S}_{\mathrm{c}}^{2}$.

We do not have a mathematical analysis of the above algorithm, since such an analysis would depend on the nature of the underlying distributions. We have no exact knowledge of the distributions, but our observations suggest that for infant subjects, the data can be modeled by a Gaussian distribution confounded with a rectangular distribution; unfortunately, such profiles are analytically intractable. Instead, we have investigated our algorithm extensively by using Monte Carlo and numerical integration techniques. For the Monte Carlo tests, a large set of simulated data was created from Gaussian fixational distributions and rectangular distributions. Provided the conditions stated earlier were met, the procedure converged satisfactorily onto the known fixation mean, usually in less than 20 iterations. For numerical integration (of series expansions), data sets were simulated from pairs of Gaussian distributions, thus providing a more demanding test of the algorithm. Some typical results illustrating the effectiveness of the algorithm are shown in Figure 3. For convenience, the fixation distribution was standardized to have zero mean and unity variance; the mean of the confounding distribution was allowed to vary and is shown along the abscissa in units of the SD of the fixation distribution (z scores). The parameter on the curves is the SD of the confounding distribution. For each simulated data set, the value of the final mean, $\bar{T}_{1 a s t}$, is shown on the ordinate, also as a 2 score. As an example, consider a confounding mean of 2.5: If the SD of the confounding distribution is 1.0 (i.e., equal to that of the fixational distribution), then the derived best estimate of the fixation mean, $\overline{\mathrm{T}}_{\text {last }}$, will be .1 SD away from the true mean $\bar{F}$; if the confounding SD is 5.0 , the point of convergence will be about $.5 \mathrm{SD}$ from $\overline{\mathrm{F}}$, and so on. How closely the algorithm converges on $\bar{F}$ depends on the precise details of the underlying distributions. However, in all our tests, the algorithm converged to a point well within $1 \mathrm{SD}$ of $\overline{\mathrm{F}}$. These convergence points can be compared with the dashed line denoting the values of $\bar{T}_{1}$, the initial mean of the simulated data set. The values of $\bar{T}_{1}$ are the "best estimates" that would have been obtained were all the data points considered equally representative of fixation. The results in Figure 3 were obtained using a preset rejection criterion, $\mathrm{k}=2.0$. Further numerical analyses and Monte Carlo studies have shown this value to yield the most stable convergence. 


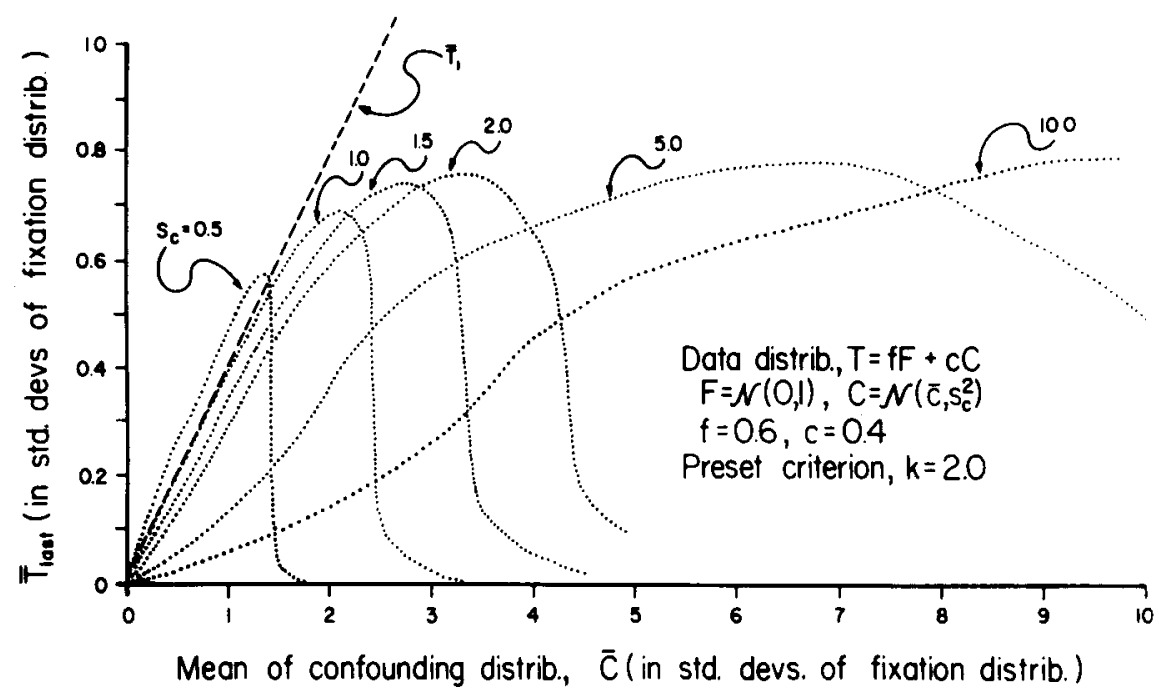

Figure 3. Simulated test of algorithm to find central tendency of fixation; numerical integration techniques were used to find convergence estimate $\left(\overline{\mathrm{T}}_{\text {last }}\right)$. For each curve, the assumed fixation distribution is Gaussian with zero mean and unity variance, $N(0,1)$; the assumed confounding distribution is Gaussian with mean $\overline{\mathrm{C}}$ and standard deviation $S_{c}, N\left(\bar{C}, S_{c}^{2}\right)$. All units are standard deviations of the fixational distribution.

This iterative procedure is applied to the calibration data by treating the data sets of each calibration point separately. Since the data are two-dimensional, the interval of acceptance (i.e., nonrejection), as prescribed by the preset number of SDs, $k$, is actually an area. In the particular case of equal SDs in $\mathrm{X}$ and $\mathrm{Y}$, this area would be circular; however, since in general the $X$ and $Y$ SDs will be unequal, the area of acceptance will become an ellipse. Thus, although the $\mathrm{X}$ and $\mathrm{Y}$ means and variances may be calculated separately, they must be combined to calculate the elliptic rejection boundary (see Figure 4).

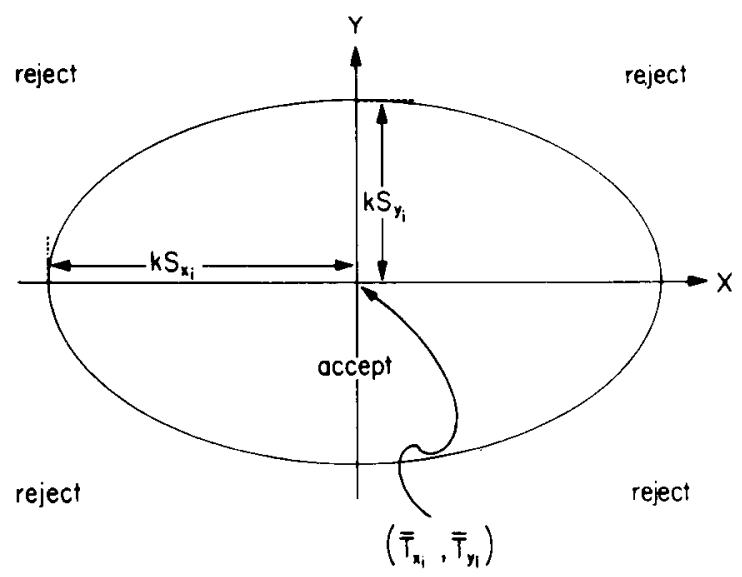

$$
\begin{array}{ll}
\frac{X^{2}}{S_{x_{i}}^{2}}+\frac{Y^{2}}{S_{y_{i}}^{2}}>k^{2} & \text { reject } \\
\frac{X^{2}}{S_{x_{i}}^{2}}+\frac{Y^{2}}{S_{y_{i}}^{2}} \leq k^{2} & \text { accept }
\end{array}
$$

Figure 4. The elliptic rejection boundary on $X$ and $Y$ for a preset criterion of $\mathbf{k}$ standard deviations.

\section{OBTAINING THE POLYNOMIAL}

From the previous iteration procedure, we obtain fixation estimates, each of which is associated with a calibration point of known absolute coordinates. The problem is to map the set of fixation estimates onto the set of calibration points by solving a two-dimensional polynomial. This polynomial is obtained by solving a set of simultaneous equations obtained from the fixation estimates and the absolute calibration coordinates. The procedure for solving these equations uses the standard least squares method (e.g., Carmody, Kundel, \& Nodine, 1980 ), with the addition that the polynomial is weighted according to the reciprocal sample variance of each fixational estimate. These reciprocal variances are incorporated to reflect the differences in reliability of the fixational estimates for the various points.

The polynomial is calculated as follows. Let: $x_{i}, y_{i}=$ the coordinate of the ith fixation estimate; $X_{i} Y_{i}=$ the coordinate of the ith calibration point; $S_{i}^{2}(x), S_{i}^{2}(y)=$ variance of the ith fixation estimate obtained from the unrejected points on the last iteration for the ith point; $\mathrm{n}_{\mathrm{i}}=$ number of remaining points after the last iteration of the ith point; $M=$ number of calibration points; $w_{i}(x)=n_{i} / S_{i}^{2}(x), w_{i}(y)=n_{i} / S_{i}^{2}(y)=$ weighting factor for the ith fixation estimate; and $\mathrm{i}=1, \mathrm{M}$. By separating the coordinate dimensions, we now prescribe the following polynomials:

$$
\begin{aligned}
& X_{i}=a_{1}+a_{2} x_{i}+a_{3} y_{i}+a_{4} s_{i}^{2}+a_{5} y_{i}^{2}+a_{6} x_{i} y_{i} \ldots \\
& Y_{i}=b_{1}+b_{2} y_{i}+b_{3} x_{i}+b_{4} y_{i}^{2}+b_{5} x_{i}^{2}+b_{6} x_{i} y_{i} \ldots
\end{aligned}
$$

We can solve for $M$ coefficients for both polynomials by constructing a total square error term (Equation 2) in the usual fashion except for the variance-dependent 
weighting factor, $\mathrm{w}_{\mathrm{i}}$; we can minimize this error by setting the derivative with respect to each coefficient to zero, as shown below in Equations 3a-3f (for simplicity, only the equations in $\mathrm{x}$ are shown).

$$
\begin{gathered}
e_{x}^{2}=\sum_{i=1}^{m} w_{i}(x)\left[X_{i}-\left(a_{1}+a_{2} x_{i}+a_{3} y_{i}+\ldots\right)\right]^{2} \\
\frac{\partial\left(e_{x}^{2}\right)}{\partial a_{1}}=-2 \sum_{i=1}^{m} w_{i}(x)\left[x_{i}-\left(a_{1}+a_{2} x_{i}+a_{3} y_{i}+\ldots\right)\right]=0 \\
\cdot \cdot \\
\cdot \\
\frac{\partial\left(e_{x}^{2}\right)}{\partial a_{6}}=-2 \sum_{i=1}^{m} w_{i}(x)\left[x_{i}-\left(a_{1}+a_{2} x_{i}+a_{3} y_{i}+\ldots\right)\right] x_{i} y_{i}=0
\end{gathered}
$$

The simultaneous equations, $3 \mathrm{a}-3 \mathrm{f}$, can be solved by any standard method (e.g., matrix inversion). Only six coefficients are considered, but, of course, up to $\mathrm{M}$ may be used if necessary.

Once the coefficients $a_{j}, b_{j}(j=1, M)$ have been evaluated, it is a simple matter to calibrate the experimental data. Each experimental point's coordinates are substituted for $x_{i}, y_{i}$ in Equations $1 a$ and $1 b$ to obtain the corrected values.

The maximum number of coefficients in Equations $1 \mathrm{a}$ and $1 \mathrm{~b}$ is, of course, equal to the number of calibration points for which fixation estimates were obtained. Since one cannot guarantee obtaining a full set, it is important to consider the order in which points are presented to the subject and the meaning of each coefficient. In Equation $1,\left(a_{1}, b_{1}\right)$ represent constant displacement errors, $\left(a_{2}, b_{2}\right)$ are linear magnification terms, $\left(a_{3}, b_{3}\right)$ are linear rotation terms, $\left(a_{4}, b_{4}\right)$ are quadratic displacement terms possibly due to optical spherical aberration, and so on, through the remaining second-order corrections. On the assumption that infants may complete only part of the full calibration series, we suggest that the order of presentation maximize the area of the stimulus screen that is covered; for example, a satisfactory order might be like the one shown in Figure 1. The absence of data for unattended calibration points effectively reduces the order of the available polynomial, and, consequently, less error can be corrected. However, the algorithm has been found to be quite robust for as low as three calibration points.

\section{CORRECTING EXPERIMENTAL DATA}

The application of this algorithm can best be illustrated by considering actual data. Figure 5 a shows a plot of calibration data for an infant subject viewing a flashing calibration point. As can be seen, the subject seems to be looking to the left of the calibration point; the source of this error is, of course, unknown. Similar data are collected for as many of the remaining calibration points as possible. The coordinates for the data for

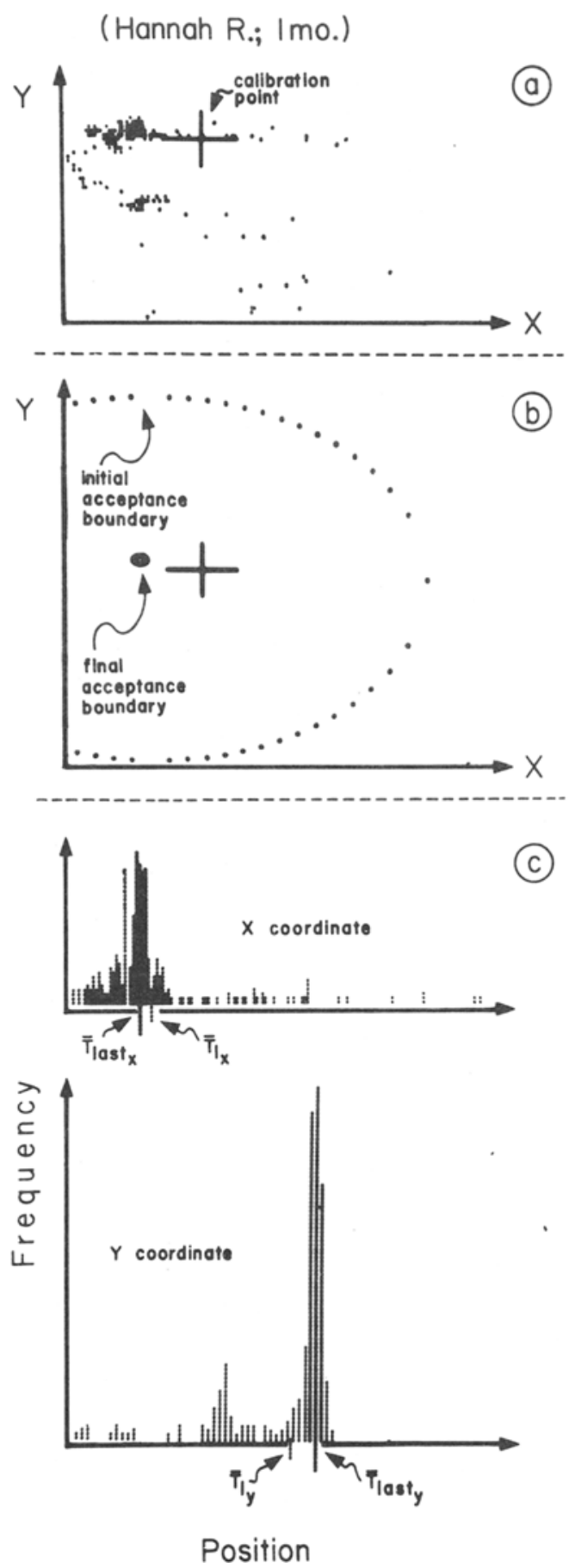

Figure 5. (a) A graphic representation of actual calibration data obtained from an infant subject viewing a calibration point (the cross). (b) Rejection boundaries for the first and the last iterations on the data shown in Figure 5a. (c) The frequency distributions for $\mathrm{X}$ and $\mathrm{Y}$ of the data plotted in Figure 5a.

this calibration point are held in a buffer in core (having been read in from the mass storage device) for the iterative procedure to find the best estimate of the subject's fixation point. The outer ellipse in Figure $5 \mathrm{~b}$ shows the extent of the very first acceptance area; only 

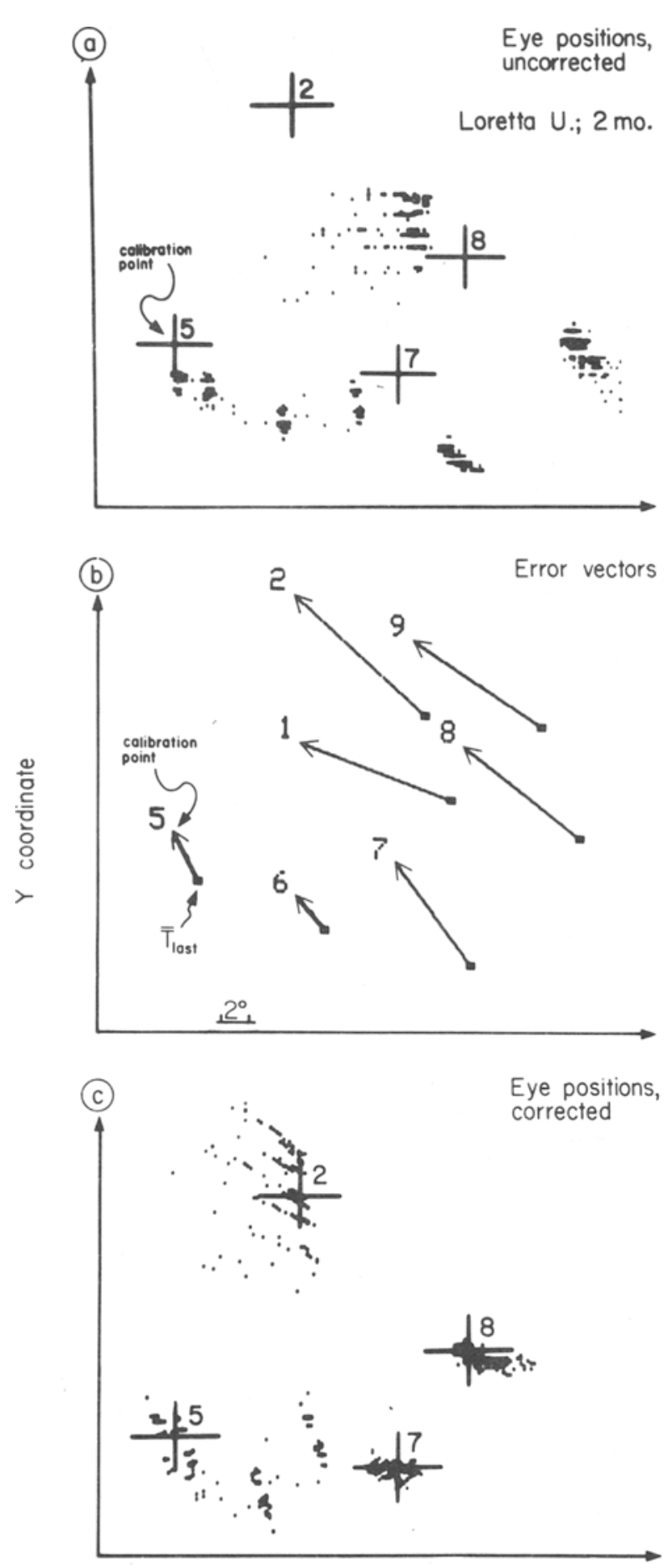

\section{$X$ coordinate}

Figure 6. (a) Data for a set of calibration points (crosses). (b) The lines indicate the error vectors between each calibration point and its associated fixational estimate (block). (c) The effect of applying the polynomial to the original calibration points. (Note-The numbers indicating the calibration points do not correspond to the preferred sequence in Figure 1.)
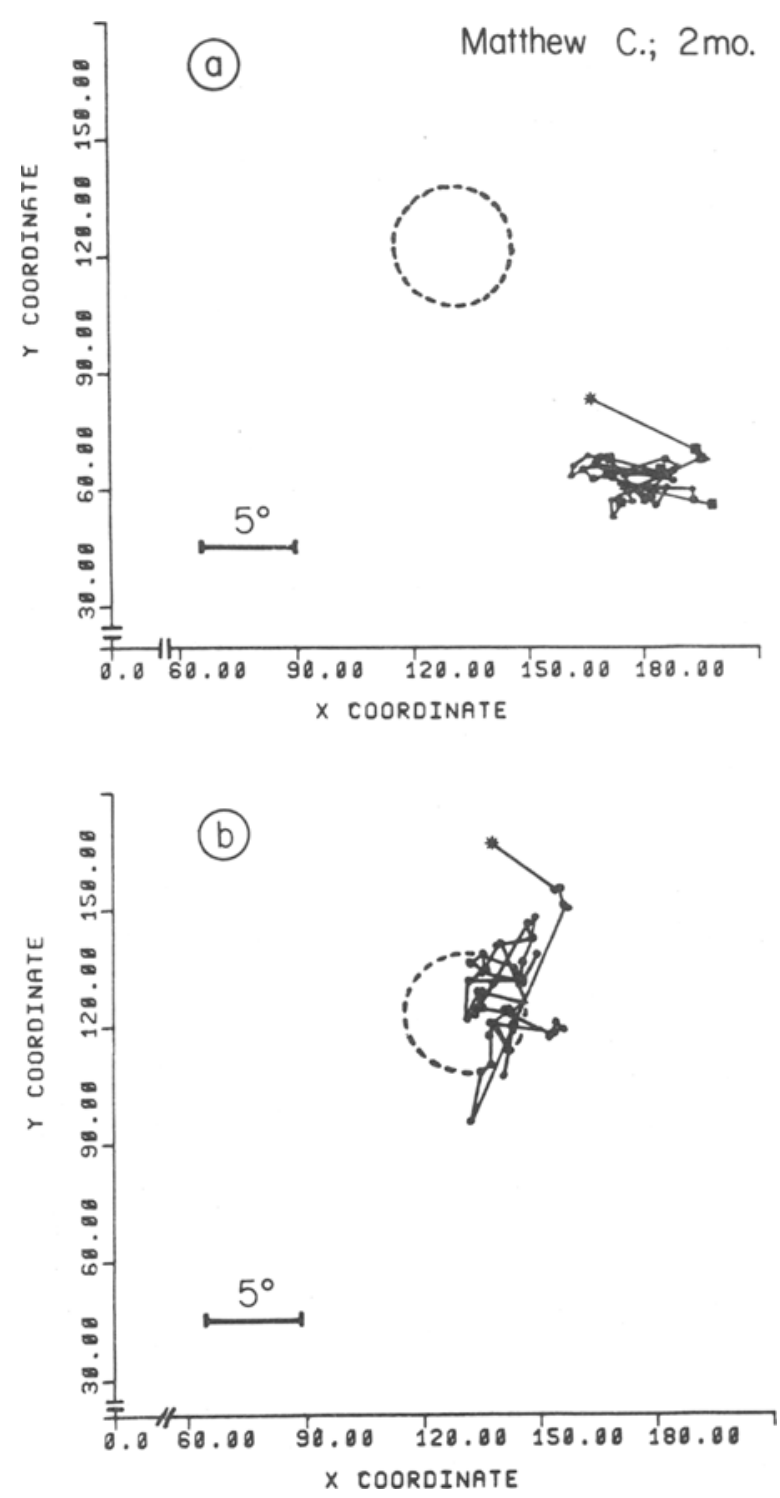

Figure 7. An example of the effect of applying a calibration polynomial to experimental data from an infant viewing a 5-deg circle. (a) Uncorrected fixation data. (b) Data corrected by the subject's calibration polynomial.

points lying within this ellipse are used to calculate the means and variances for the next iteration. The process continues until it converges to the inner ellipse, the center of which represents the final mean $\overline{\mathrm{T}}_{\text {last }}$, the best estimate of the subject's point of fixation. As can be seen, the highly deviant points have been successfully rejected. Figure $5 \mathrm{c}$ shows the distributions of the raw data in the $\mathrm{X}$ and $\mathrm{Y}$ directions.

When the points of fixation for all the calibration points have been found, the algorithm enters the second stage: calculating the polynomial. Figure 6a shows the calibration points and their corresponding calibration data for another infant subject (only a few of the set 
are shown, for the sake of clarity). The error vectors shown in Figure $6 \mathrm{~b}$ are used to calculate the polynomial. The resultant polynomial would normally be used to correct experimental data; however, Figure $6 \mathrm{c}$ shows the effect of correcting calibration data. As can be seen, the fixation data appear to be mapped onto the calibration points. We can never know with certainty whether a noninstructable subject is fixating the stimulus points. However, for adults, whose variability is much less, we have found the fit to be convincing.

As stated earlier, the calibration procedure is carried out off-line, which allows the analysis to be programmed on a computer larger than the one controlling data acquisition. The algorithm is written in FORTRAN IV, and it will run successfully on a PDP-8/e (Digital Equipment Corporation) with $24 \mathrm{~K}$ of memory, a floatingpoint processor, and a display oscilloscope. The major allocation of core is for a buffer to hold the calibration data.

As claimed at the outset, this calibration procedure was developed primarily for correcting data from noninstructable infant subjects. Figure 7a shows uncorrected experimental data obtained from a 3-month-old infant viewing a circle. The infant was later calibrated as outlined above to yield the polynomial: $X_{i}=416.06+$ $7.08 x_{i}-14.09 y_{i}-.03 x_{i}^{2}-.07 y_{i}^{2} ; Y_{i}=-195.72+$ $4.12 y_{i}+1.41 x_{i}-.016 y_{i}^{2}-.004 x_{i}^{2}$. Only five coefficients could be evaluated because the infant fell asleep after the fifth calibration point. The result of applying this polynomial to the experimental data set is shown in Figure $7 \mathrm{~b}$; the coincidence between corrected fixations and the stimulus is compelling, even though data from all of the calibration points were not available.

This technique offers the possibility for correcting scanning data from an infant by that infant's own calibration function. Further, by investigating the magnitudes and types of errors across infants, it can provide a "worst-case" estimate of the accuracy of eye position data from infants for whom no calibration data are available. Finally, comparison of the calibration polynomials across age may yield information about developmental changes in the functioning of the visual and oculomotor systems in early infancy.

\section{REFERENCE NOTE}

1. Abramov, 1., Gordon, J., Hendrickson, A., Hainline, L., Dobson, M. V., \& LaBossiere, E. The postnatal development of the infant retina. Paper presented at the meeting of the Society for Research in Child Development, Boston, Mass., April 2-5, 1981.

\section{REFERENCES}

Bronson, G. The postnatal growth of visual capacity. Child Development, 1974, 45, 873-890.

Carmody, D. P., Kundel, H. L., \& Nodine, C. F. Performance of a computer system for recording eye fixations using limbus reflection. Behavior Research Methods \& Instrumentation, $1980,12,63-66$.

Carpenter, R. H. S. Movements of the eyes. London: Pion, 1980.

Hainline, L. An automated eye movement recording system for use with human infants. Behavior Research Methods \& Instrumentation, 1981, 13, 20-24.

MANN, I. The development of the human eye. London: British Medical Associates, 1964.

Slater, A. M., \& Findlay, J. M. The measure of fixation position in the newborn baby. Journal of Experimental Child Psy. chology, 1972, 14, 349-364

Young, L. R., \& Sheena, D. Survey of eye movement recording methods. Behavior Research Methods \& Instrumentation, 1975. 1, 397-429.

\section{NOTE}

1. While infants will attend to the flickering lights, we are developing an alternative, possibly more "attractive" display in which the infant is presented dots that move across the screen to encourage following. The dots are then stopped at designated coordinates for data collection, as above.

(Received for publication June 23, 1980; revision accepted February 24,1981 .) 